\title{
The Social Reading Model with Virtual and Actual Combination
}

\author{
Yu Yonghai, Yu Lingling, Zhu Jihong \\ School of Mechanical Engineering, Zhejiang University of Technology
}

Hangzhou, Zhejiang

\begin{abstract}
:
social reading is a brand new reading mode which focuses on the reader and emphasizes on such social activities as sharing, interaction and communication. Born out of the booming social network, social reading can optimize the reading value and render the reading as a more effective and social activity. This paper has analyzed the social features of traditional reading and modern digital reading and concluded two different interaction modes. A social reading combining the virtual and actual features is put forward based on the previous comparison of the weaknesses and strengths of virtual and actual socialization, and the socialization relations in the two kinds of socialization.
\end{abstract}

Key words: social reading virtual actual socialization

\section{Foreword}

With the rapid development of internet at this age, social reading products come into being one after another but their interfaces and functions are seldom varied. The social function of these products is confined to the socialization in the virtual network, and they ignore the building of a sound cultural atmosphere for the reading activity and also the declining trust between people in the cyber world. Prospectively speaking, such shortcoming is unfavorable to the improvement of our socializing ability and the demand of self realization. Therefore, in order to offer a better experience to the readership, it's badly needed to advocate an active, efficient and healthy social reading mode.

\section{Social Reading from Paper to Digital}

Social reading refers to a new kind of reading mode with the reader as the center and social activities like sharing, interaction and communication as the focus. In contrast with the traditional reading mode which stresses the book and its content, social reading emphasizes the reader and the socialization of reading, advocates the creation of general user generated content (UGC), general communication and general profit, and optimizes the reading value based on the interaction between different participants like the interaction between readers and between the reader and the writer.

\subsection{Shift of interaction at different ages}

From paper media age to mobile internet age, the readers witnessed a changing interaction mode in social reading and a richer social communication channels (see Figure 1). Different from the past face-to-face vocal communication and the communication by letter at the paper media age, the social communications like social networking, blog and personal websites have lifted the time and spatial restrictions in the communication and make communication available anytime and anywhere. 


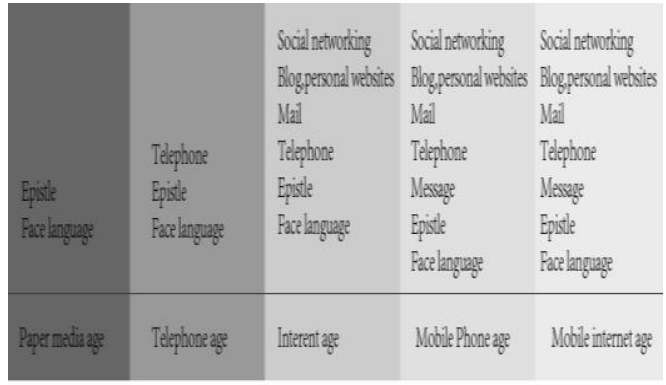

Figure 1: The Shift of

Interactions at Different Ages

\subsection{Interaction experience in the traditional book-centered reading}

The interaction in the social reading in the form of traditional paper is linear and singular. The reading activity is represented by the circulated reading between good friends who recommend or borrow the book he has read to his friends who, after a reading, exchange their readership opinions. A group reading activity may also be organized or occur accidentally which is a more active and dynamic experience. The Figure 2 below betrays the book-centered interaction process.

\section{"book" $\longrightarrow$ Borrow/share reading. $\longrightarrow$ exchange}

Figure 2: Book-Centered Simple Interaction

\subsection{Interaction experience in the user-centered digital reading}

The interaction amongst the social reading in digital space is rich in forms. Nowadays, the digital reading has seen the flourishing of different reading appliances and the diversification of the book acquirement ways for the readers who can enjoy a convenient and efficient online communication. The social activities out of readership's reading needs mainly include their evaluation and recommendation of the content they have read, the communication between the reader and the writer and the automatically introduced content for reading.
All of these communication activities can be undertaken just by a simple click or input on the mobile appliance. Through the computer's intelligent screening technology, the reader can find the content he wants more easily and so the social reading shifts from the book-centered to user-centered style (Figure 3).

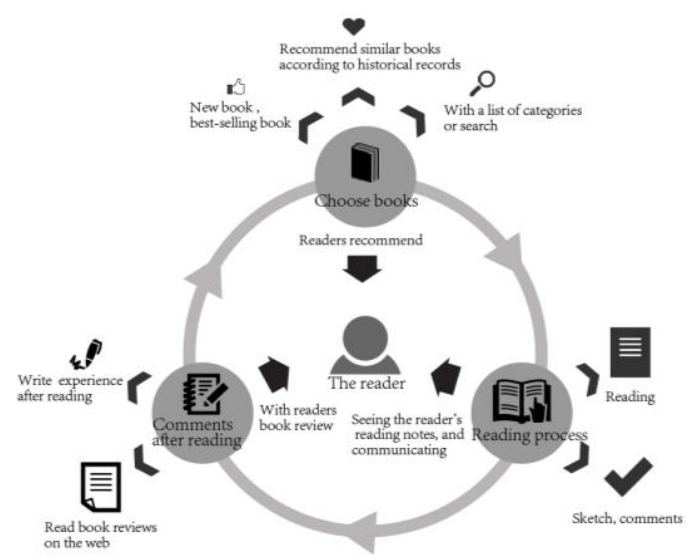

Figure 3: Rich Interactions in the Mobile Internet

The analysis of the interaction modes of traditional and digital reading reveals that the basic manners of both social reading are similar and can be generally classified into the two kinds of social behaviors, sharing and recommendation, evaluation and communication.

The various interaction forms of both traditional and digital readings are the extension of reading activity per se. The difference of the two social readings is the communication ways, but the essence of the socialization behavior behind them is not much different. The shift from book-centered social experience to user-centered social experience makes the social communication more humanized. With the change of socializing method in the form of the initial face-to-face manner and the present mobile network, the approaches of social communication become more and more diversified. Such social communication behaviors in the paper and 
digital reading space are born along with people's reading activity. They offer us more information for reading and enrich our reading life.

\section{The Analysis of Actual and Virtual Socialization in Social Reading}

\subsection{Complementary advantages of social communication}

In terms of reading activity, both the actual socialization in the traditional paper reading and the virtual socialization in the digital reading have positive and negative influences.

The socialization in the traditional book-centered reading offers the reader a sound reading atmosphere and the face-to-face communication also enriches their reading life. However, this kind of direct communication also has its own limitations or internal features: the privacy of the communication environment (enclosed space), the pertinence of communication subject (acquaintance) and the transience of communication content (oral information) [1], whose objective and subjective conditions restrict the social communication in the traditional reading. For instance, readers can hardly choose a place and time all feasible for them; it's difficult for them to find other readers to share their reading; they can seldom make notes on the book he has borrowed and so may forget many valuable details. Besides, since it's linear and singular, the transmission content is absolutely controlled by the transmitter, resulting in the absence of feedback and interaction from readers, though the readers may sometimes make their own choice, which actually is under the control of the transmitter and media, about the medium and reading material according to their interest and habit.

The socialization in the mobile user-centered digital reading changes the single information communication model in traditional reading, mainly reflected by the improvement of interaction between subject and object, the involvement of readers in reading and feedback, and the role transformation from information receiver to provider. It has been found that the information exchange is the main content of interaction among members of virtual community including the search, provision and sharing of information ${ }^{[2]}$. But due to its scare restriction of information releasing, the high availability of massive resources, the non-boundary and flexibility of communication and the virtual identity of participants, readers also have to receive unwanted information while indulged in the experience of digital reading. Therefore, they have to spend more time in identifying the value and credibility of the information which increases their reading burden. The confidentiality of virtual socialization also causes some social problems: first, the uncertainty of information in the virtual socialization leads to the distrust to each other; second, the readers may conduct some virtual activities harmful to socialization. Also, the overreliance on the virtual socialization and the indulgence in the virtual world not only weakens our communication ability in the outside actual world but also do harms on our health.

The social communication in the virtual digital space has overcome the weaknesses of singular, linear social communication in traditional reading and enriches the content of socialization. By contrast, the face-to-face communication in traditional reading builds a good atmosphere. So either the paper or digital reading is significant by existence and is an effective way for readers to acquire more reading resources. They are both the outcome of the times and will persist for good, free from any elimination for their 
weakness.

\subsection{The link of social relations}

American socialist Granovetter divided interpersonal network into strong relation network and weak relation network. The former means that personal social network is very homogeneous, the social resources are similar and interpersonal relation is maintained by a strong emotional factors. On the contrary, the weak relation network is featured by a strong hetorogeneity in personal social network, divergent social resources, loose human relation and scarce emotion maintenence. The strengh of relation determines the nature of the information acquired and the possibility for an individual to achieve his objective. In Granovetter's study, weak relation refers to those relations shallow and unfrequent. But it can offer"information and resource channels for people to transcend the social community they belong to". From the perspective of the mainstream forms of social network in China, Chinese people are gradually shifting from a strong to a weak relation, which actually stems from their overreliance on social networking.

From the perspective of social reading, the actual socialization in traditional reading exhibits the strong relation pattern of interpersonal relation network. The establishment of a strong relation for someone is closely related to his social environment. Take a student for example. He has many student friends whose identities, backgrounds and educations are highly identical, whose human resource is about the same and whose mutual trust is strong. The features of personal identity limits our social circle. The virtual socialization in digital reading, on the contrary, exhibits a weak relation pattern which determines that the human relation in this reading community is not as close as that in the socialization of strong relations. In the reading community of a book or a class of books may be school students or white-collar workers. So in such weak relation, soical resources are abundant and heterogeneous. When the weak relation serves as an effective bridge of social relations to connect the communication circles of humans, it not only can expand our social circle but aslo help us make more friends and increase our knowledge.

\subsection{The socialization pattern of Douban}

\section{City-site: "Network-Reality-Network"}

The offline activity of Douban City-site is a good example of the combination of virtual and actual socialization. Its major operation mode is a social network of city community with online initiation of and offline participation of activities.

The mode can be divided into four stages: 1. An individual or organization initiates a group activity such as watching performance and visiting an exhibition;

2. Internet users choose relevant activity in the local city site according to his interest and obtain such information as activity time, place and requirements;

3. The initiator of the activity arranges participants to carry out activities offline or in reality;

4. After the activity, the participants discuss and share their feelings on the website.

The most critical part of the whole process is undertaken in the reality while the network only assumes the preparation and follow-up. Different from the transmission models of Network to Network in virtual socialization and of reality to reality in actual socialization, this kind of transmission model can be generalized as Network to Reality and to Network.

Douban City-site integrates the virtual community and the actual life whereby the information channel is expanded through the strong heterogeneity of social network 
generated by the weak relation of virtual community and the strong trust and authenticity brought by the strong relation of the actual socialization. Such a kind of combined virtual and actual pattern somewhat avoids the shallow communication common in the weak relation and the restriction of socialization content and subject in the strong relation. Thereafter, the activity's degree of freedom will be optimized, the effectiveness and authenticity of the information will be improved, the influence of the activity will be strengthened and the social network will be persisted. That's also why Douban City-site is distinguished from other virtual community and favored by so many young adults.

\section{Social Reading Mode with Virtual and Actual Combination}

Considering the respective weakness and strength of actual and virtual socialization in the social reading, the combination of the two reading modes will make social reading more penetrating. In reference to the information circulation mode of Network to Reality and to Network in Douban City-site, we propose a social reading mode combining virtual and actual features. Such interactive mode can be concluded as follows: (see Figure 4)

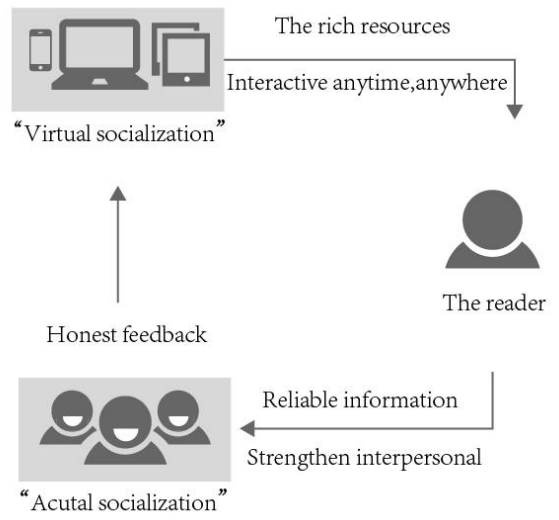

Figure 4: social reading mode with virtual and actual combination
The social interactive mode with virtual and actual combination is characterized by:

1. Readers initiate reading activities based on the rich resources in virtual socialization;

2. Readers participate in the activity offline and communicate with each other face to face;

3. Readers return to the virtual internet to discuss and share the activity.

Accroding to their appetite and interest of book as well as their reading content and mode, the users can be classified into different groups with different identities and resources, creatng more plentiful conditions for acutal socialization. Thanks to the disclosedness and authenticity of information, the content and itneraction in the social reading is postively correlated. In the social reading, the interaction between readers and between the reader and the writer can be commented and feedbacked by users. With the reality of actual socialization and the effeciency, freedom and promptness of virtual socialization, the combination of virtual interaction and acutal activity can help build a stronger connection between the individual and the group. This combined social reading will exercise a stronger influence and has effectively avoided the weakness of singular socialization mode. The social reading community with the objective of book reading builds a pretty good cultural atmosphere for our life, relieves the fatigue of our internet life and improves our socializing ability.

\section{References:}

[1] Huang Minxue, Wang Yan, Jiang Shuqin, "On the Virus-style Interpersonal Information Communication Mechanism on the Internet", Luojia Management Review, Vol. 1, 2011

[2] Burnett GInformation exchange in 
virtual Communities : A

Typology.[J]Information Research, Vol.5

No.4,July 2000.

[3] Zhang Siqi, Weibo Culture Study, Jilin University, March 8, 2013

[4] Wang Meng, "Changes and Actual

Return of Virtual Community

Communication Mode-A Case Study on

Douban City-site", Jilin Radio and TV

University Journal, Vol.2, 2012 